\title{
Investigating the Impact of Dwell Time on the Reliability of Urban Light Rail Operations
}

\author{
Zoi Christoforou $^{1} \cdot$ Ektoras Chandakas $^{2} \cdot{\text { Ioannis } \text { Kaparias }^{3} \text { (D) }}$
}

Received: 9 August 2019/Revised: 20 December 2019/Accepted: 19 February 2020/Published online: 19 March 2020

(C) The Author(s) 2020

\begin{abstract}
The present study investigates the determinants of vehicle dwell time at stations in urban light rail networks. Using data collected from an on-board automatic passenger counting system of the tramway network of the French city of Nantes over a long period, the study performs graphical and statistical analyses enabling the identification of cause-and-effect relationships of a number of attributes on the dwell time and its reliability. The results confirm the significance of the boarding and alighting passenger volumes, as well as of the on-board passenger loading, on the dwell time. Additional effects on dwell time are found from the vehicle type (low- or high-floor), the time of day (peak, off-peak, inter-peak) and the location of the station (city centre, proximity to points of interest).
\end{abstract}

Parts of this paper were previously presented at the 95th Annual Meeting of the Transportation Research Board and at the 8th International Congress on Transportation Research in Greece.

Ioannis Kaparias

i.kaparias@southampton.ac.uk

Zoi Christoforou

zoi.christoforou@enpc.fr

Ektoras Chandakas

ektoras.chandakas@transamo.com

1 Laboratoire Ville Mobilité Transport, Université Paris Est, 6 et 8 Avenue Blaise Pascal, Cité Descartes, Marne-la-Vallée Cedex, 77455 Paris, France

2 Transamo, Transdev Group, 21 Camille Desmoulins Street, Issy-les-Moulineaux Cedex, 72789 Paris, France

3 Transportation Research Group, Faculty of Engineering and the Environment, University of Southampton, Boldrewood Innovation Campus, Building 176, Room 4061, Southampton SO16 7QF, UK

Communicated by Xuesong Zhou.
Also, it is found that operations are not symmetrical, and dwell times tend to be higher in one direction than the other of the same line. Finally, the results suggest that dwell time reliability is lower for stations located further from the starting terminal, or for stations located in the city centre.

Keywords Dwell time - Travel time reliability - Urban light rail · Graphical analysis $\cdot$ Multiple linear regression

\section{Introduction}

The fact that travel time in transport networks is not constant, but entails an element of variability, resulting in uncertainty when attempting to predict it, has long been recognised in the literature [1]. The importance of this uncertainty for travellers and operators alike has been investigated extensively over the past few decades (e.g. [2-4]), and much research has been carried out attempting to quantify it through appropriate reliability measures, concentrating predominantly on road traffic. As opposed to road networks, however, where traffic congestion can be fairly easily identified as the sole source of uncertainty, passengers in public transport networks of large cities may be exposed to delays arising from multiple different sources. For instance, a study by Turnquist and Bowman [5] focused on bus networks and identified that variability existed primarily in the in-vehicle time (as the impact of traffic congestion on the bus), but also in the out-of-vehicle time (i.e. time spent waiting or transferring), which may be prone to the effects of aspects such as service instability and overcrowding. The same applies to urban rail networks, but there the in-vehicle time variability is generally less pronounced than it is in bus networks (as there is only limited interaction with road traffic, if any, and delays are 
almost exclusively the result of operational incidents), and the out-of-vehicle time variability occupies a more prominent place. Such variability can have a significant impact when it comes to the implementation of advanced operational strategies such as timetable synchronisation and optimisation or train skip-stopping [6,7].

An important component of travel time in urban rail networks is the dwell time of vehicles at stations. Its significance may not be immediately obvious when considered at the level of an individual station, as the dwell time there is usually only a few seconds long and rarely exceeds 2 min under normal circumstances. However, when accumulated along an entire vehicle run or passenger trip, it can amount to a fairly significant proportion of the total journey time. For instance, preliminary analysis by the authors using data from various light rail lines in different cities in France (namely, Le Havre, Rouen, Saint Etienne and Nantes) found that the total dwell time amounts to as much as $25-30 \%$ of a vehicle run's total journey time. What is even more important is that dwell time can be a source of variability in the total travel time, over which the operator may have little or no control and influence. This variability may be further amplified downstream the line, resulting in the so-called bunching problem. In addition, given that dwell time is in essence idle time rather than actual travel time, it is not seen favourably by travellers and can negatively affect their perception of customer service.

But while dwell time and its variability are recognised as important influencing factors of the operation of large cities' urban rail networks, its causes and potential effects have received relatively little attention in the literature, and rail operators in most cases opt to estimate it using nonvalidated fixed-value assumptions or basic rule-of-thumb empirical techniques. The aim of this study is, hence, to shed light into the under-explored topic of vehicle dwell time at urban rail facilities by investigating not just how dwell time is influenced by different parameters, but also how its reliability (i.e. the extent of its variability) is affected. In so doing, the study seeks to identify cause-andeffect relationships through which operators could devise solutions for minimising this important source of time loss. Focusing on light rail networks, and specifically on the tramway of the French city of Nantes, and using data obtained from on-board measurements, the study investigates dwell time and dwell time reliability patterns through a graphical analysis in the first instance, and then derives statistical models enabling prediction as a function of a number of other factors.

The paper is structured as follows: Section 2 presents the background of the study, focusing primarily on previous research on the topics of dwell time and travel time reliability analysis and modelling. Section 3 then goes on to present the study site, the data collection method employed, and the analysis methodology. Section 4 reports the results of the graphical and statistical analysis performed, and presents models for explaining and predicting dwell time and dwell time reliability. Finally, Section 5 concludes the paper and identifies areas of future research.

\section{Background}

\subsection{Modelling of Dwell Time}

The understanding of the determinants of dwell time and its modelling has attracted significant attention from the scientific community. Dwell time is understood as the period during which a vehicle is immobilised at a station. It comprises three components: the door manoeuvre time (door opening and closing) and vehicle departure, the passenger flow time, and the time the doors remain open without passenger flow [7]. Therefore, a link exists between passenger flows (defined as the total number of boarding and alighting per service or vehicle) and service operations, where the former influences the latter and vice versa.

The transit capacity and quality of service manual (TCQSM) of the US Transportation Research Board (TRB) [8] proposes a function of boarding and alighting flows for the calculation of a vehicle's average dwell time:

$\mathrm{DT}=x_{\mathrm{a}} t_{\mathrm{a}}+x_{\mathrm{b}} t_{\mathrm{b}}+t_{\mathrm{c}}$

where $x_{\mathrm{a}}$ and $x_{\mathrm{b}}$ denote the alighting and boarding passenger numbers through the busiest door, while the parameters $t_{\mathrm{a}}$ and $t_{\mathrm{b}}$ express the service time per passenger for boarding and alighting, respectively (i.e. the time that it takes for a passenger on average to complete a boarding or alighting process), and $t_{\mathrm{c}}$ corresponds to the time needed for the doors to open and close, and for the vehicle to depart.

While Eq. 1 is widely used by public transport practitioners due to its simplicity, it does not include the particularities of different modes. This matter boasts extensive literature, with each one of the different studies focusing on a single or on a number of determining factors of dwell time by mode (bus, light rail, metro and train). For instance, Szplett and Wirasinghe [9] and Wirasinghe and Szplett [10] investigated the impact of the distribution of passengers waiting at a station, and inferred dwell time models that take the station location into account (suburbs and central business district (CBD)). Oliveira et al. [11] studied a given train station for evidence of concentrated boarding around the platform entrance and discussed their impact on dwell time. The impact of overcrowding and the difference among different vehicles was demonstrated in a study by Fritz [12]. Along the same lines, Lin and Wilson 
[13] proposed linear and non-linear dwell time models to consider the number of boarding, alighting and on-board passengers for one- and two-car vehicles. Cornet et al. [14] used a dataset acquired from automatic passenger counting (APC) and automatic vehicle locating (AVL) sensors on commuter rail to investigate the concept of minimum dwell time and estimate the conditional distribution of dwell time with respect to the passenger flows. Currie et al. [15] conducted a survey to establish a positive correlation between dwell time and on-board congestion.

A considerable amount of research has focused on assessing the impact of the interface between vehicle and platform and of the boarding process. Daamen et al. [16] established the impact of a vertical or horizontal gap on the passenger flow rates, while Fernandez et al. [17] focused on the relation between horizontal gap and door width and the impact of on-board payment. They concluded that a small gap reduces the passenger flows, and they inferred dwell time models for use in metro stations and bus stops. Both previous studies were based on laboratory experiments where it was possible to isolate and extract each of these effects separately. In another study by Milkovits [18], the impact of payment methods on the dwell time was investigated using data from automatic fare counting (AFC), APC and AVL systems installed in an urban bus system. The results suggested that smartcards can accelerate the payment process on board and can hence reduce vehicle dwell time; however, the benefits were mostly evident in uncongested conditions, as the effect was not noticeable under crowding.

In the urban rail context, Weston [19] developed a sophisticated formula for the London Underground that takes into account an important number of variables. Harris and Anderson [20] confirmed the validity of Weston's formula on other metro systems, although they agreed on the site-specific character of the passenger boarding and alighting service times. Harris [21], on the basis of an experiment conducted with train mock-ups, suggested that passenger boarding and alighting times are not constant during the dwelling process, but that they vary significantly as it evolves. Indeed, the fastest alighting times come from the first alighting passengers, while the fastest boarding times come from those in the middle of the boarding group.

An alternative approach for the modelling of dwell time comes from the use of micro-simulators. Zhang et al. [22] developed a micro-simulation model where passengers correspond to cellular automata. By defining the behaviour of the passengers against obstacles and attractions at an individual level, the simulation enables a range of complex phenomena to emerge on a macroscopic level on the platform during the vehicle's dwelling. It is then possible to understand the interactions between passengers and assess the impact of different stations, vehicle configurations and passenger flows on dwell time and its reliability.

\subsection{Quantification of Travel Time Reliability}

Many empirical studies have identified the importance of travel time reliability. In a study by Jackson and Jucker [2], it was found that there is a trade-off between travel time and reliability for travellers. In another study by Abdel-Aty et al. [3], it was demonstrated that a traveller's route choice is influenced by reliability and also by travel information when available, as this is seen as a way to reduce travel time uncertainty. A later study by Lam and Small [4] concluded that reliability is an important factor affecting route choice and distinguished between genders, showing that women tend to be more risk-averse than men and hence value reliability more.

Acknowledging the importance of reliability, many studies have attempted to incorporate it into a model, such as the work of Noland and Small [23], who identified that many travellers adopt a safety margin during their morning commute, so as to take uncertainty into account as much as possible, and applied penalties for early and late arrival. In another study by Bates et al. [24], travel time uncertainty was formulated as an additional "schedule disutility" in the traveller's journey time valuation function, proportional to the standard deviation of the travel time distribution, while a study by Liu et al. [25], formulated travellers' route choice as a mixed logit model, containing coefficients representing individual travellers' preferences towards travel time, reliability and cost. Other studies aimed at modelling travel time reliability can be found in the literature; comprehensive reviews of this topic have been carried out by Bates et al. [26] and Noland and Polak [27].

A considerable amount of research has focused on defining adequate measures for quantifying travel time reliability. Most of them use various characteristics of the travel time distribution, such that two types of measures can be identified: measures indicating the probability that a certain link is unusable, and measures attempting to quantify the amount of congestion that may be encountered on a link.

The most widely used reliability measure so far belongs to the first category and is the one defined by Bell and Iida [28], which is expressed as the probability of a link being uncongested, based on the assumption that the condition of traffic flow on a link is binary, i.e. congested or uncongested. While this measure is suitable for quantifying the reliability of a network, it has the disadvantage that it does not give any indication on the amount of delay that may be encountered. Other measures developed along the same concept are similarly limited, such as the ones developed by Chen et al. [29] and Eleftheriadou and Cui [30]. 
Thus, a number of studies have attempted to quantify reliability by using the travel time distribution directly. The first measure was adopted in a study by Polus [31], where reliability was defined as the inverse of the standard deviation of the link's travel time distribution. The main disadvantage of this measure, however, is the fact that it is not dimensionless. This is also the case of measures developed in further studies, such as Dandy and McBean [32], who used the 95th percentile travel time, and Lam and Small [4], who quantified reliability as the difference between the 90th percentile and the median of the travel time distribution. Some studies further developed reliability measures, not only considering the width of the travel time distribution, but also its skewness. For instance, van Lint and van Zuylen [33] proposed two reliability metrics, based on the 10th, 50th and 90th percentiles of the travel time distribution. Other reliability metrics address the passenger additional travel time [34] and the reliability buffer time [35].

Along the same lines, Lomax et al. [36, 37] proposed a series of measures of reliability and categorised them in three groups, as statistical range measures (relatively "unprocessed", based on concepts generally not understandable by ordinary travellers), buffer time measures (intended for travellers and indicating how much extra time should be allowed to account for uncertainty) and tardy trip indicators (representing the unreliability impacts in terms of the amount of late trips). Finally, Kaparias et al. [38] defined a measure that can be used both by travellers and by operators, consisting of two indices (earliness and lateness) and expressing the relationship between travel time and variability; defined as the ratio of the mean to the 5th percentile and of the 95th percentile to the mean respectively, the two indices enable not only the characterisation of network links as reliable or unreliable, but also the quantification of the uncertainty to be encountered in the form of lower and upper bounds. This paper focuses on the analysis of the dwell time reliability, as part of the travel time reliability of light rail operations.

\subsection{Study Contribution}

The present study builds on previous scientific contributions and, using extensive APC data on an urban light rail system, it expands the categories of variables used to estimate dwell time and its variability. Rather than limiting the explanatory variables to the passenger state flows (boarding, alighting and on-board volumes), it extends the scope of the study by additionally investigating the impact of variables relating to the station environment, such as proximity to the CBD and presence of a point of interest (POI), and to the light rail system design and operational characteristics (such as rank of the station, direction and type of rolling stock). These contributions improve the understanding on the determinants of the dwell time in an urban setting.

\section{Methodology}

\subsection{Site Description}

The present study focuses on the tramway system of the French city of Nantes. Nantes is located on the Loire river in western France, close to the Atlantic coast. It is the sixth largest city in France, with a metropolitan population of 900,000 . Its tramway network is operated by Semitan, and with its opening in 1985 Nantes became the first city to introduce a modern-generation tramway, built from scratch. Nowadays the network consists of three lines (numbered 1,2 and 3) running on $44 \mathrm{~km}$ of track and serving a total of 83 stations.

The Nantes tramway is shown in Fig. 1. Line 1, shown in green, is $18.4 \mathrm{~km}$ long and serves 34 stations. It consists of two branches at each end (Beaujoire and Ranzay in the east, and François Mitterrand and Jamet in the west) and a central trunk between the branches, with 19 stations. Its frequency reaches 15 vehicles per hour during peak times, and it is the busiest line on the network (and with 120,000 passengers per day, it is also one of the busiest in the whole of France), serving several principal locations in the city, including the city's stadium and the main railway station. Line 2, shown in red, runs from Orvault in the north to Gare de Pont Rousseau in the south, is $11.7 \mathrm{~km}$ long and serves 25 stations, including important educational (university) and health establishments. It has a frequency of eight vehicles per hour during peak times, and its patronage approaches roughly 80,000 passengers per day. Lastly, Line 3, shown in blue, runs from Marcel Paul in the north to Neustrie in the south, is $14.1 \mathrm{~km}$ long and serves 34 stations. Its operations are similar to Line 2, with which it shares the track for seven stations (Hôtel Dieu to Gare de Pont Rousseau) in the city centre. It serves several major commercial sites and is used by 75,000 passengers per day. The three lines run radially off the city centre but meet at Commerce. They are combined with park-and-ride $(\mathrm{P}+\mathrm{R})$ facilities on the outskirts, and also have major transfer points with the other public transport modes: the Busway (exclusive right-of-way bus rapid transit (BRT)), the Chronobus buses (buses with limited segregated lines), the local buses and the regional coaches.

The tramway system is served by three types of rolling stock, irrespective of the line: the Alstom Tramway Français Standard (TFS), the Bombardier Incentro, and the CAF Urbos. The Alstom TFS is a 39-m-long vehicle with a 236-passenger capacity (including 74 seats) which began 

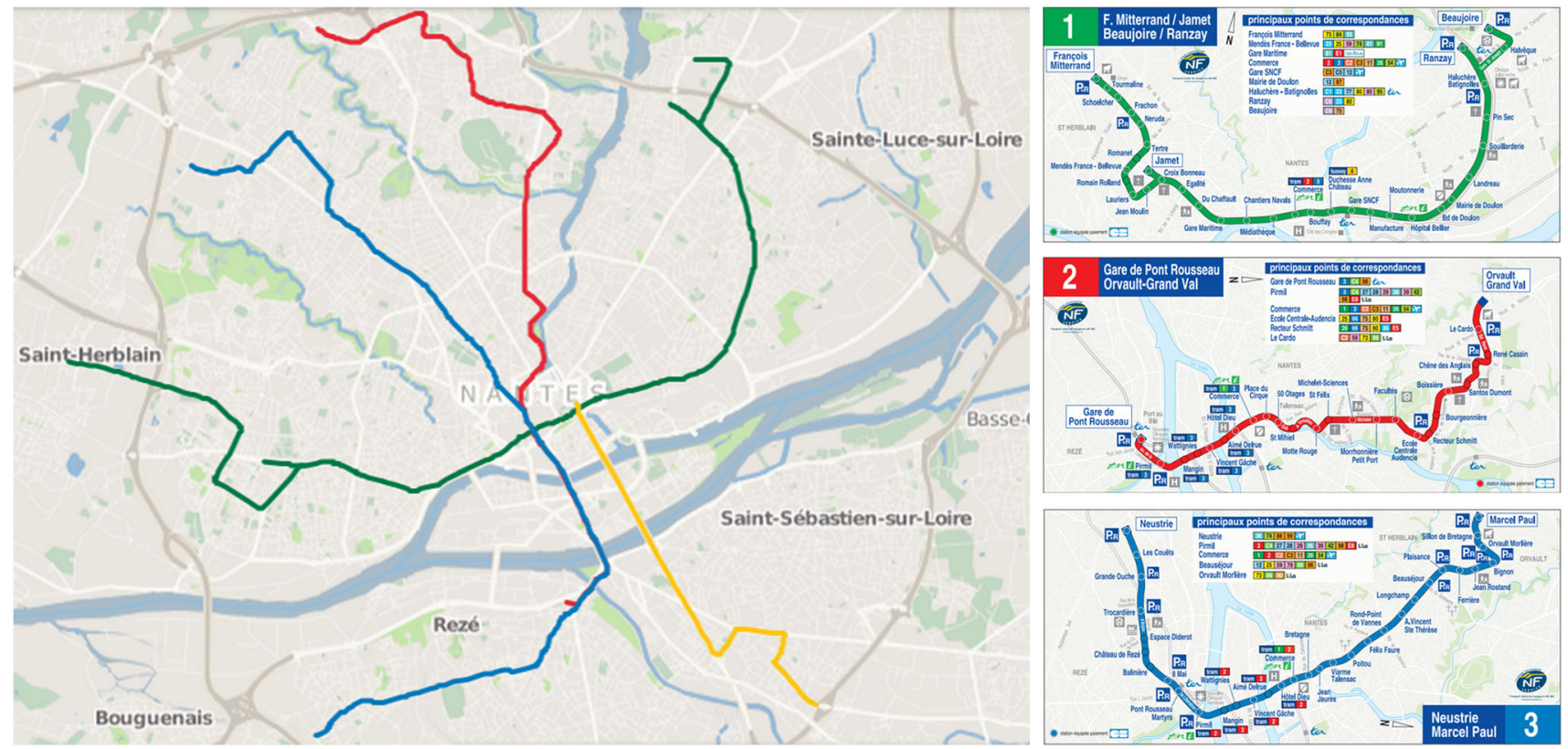

Fig. 1 The Nantes light rail network. (Source: www.tan.fr)

operations in 1985. Each Alstom vehicle is composed of two high-floor carriages with three-step access (of which one is a mobile step) and a lower-floor carriage in the middle; access is provided by six double-length doors and two simple doors per vehicle side. The Bombardier Incentro is $36 \mathrm{~m}$ long, with capacity for 252 passengers (including 72 seats), and started operating in 2000. It has an integral low floor and six double $(1.30 \mathrm{~m})$ doors per side. Finally, the CAF Urbos is the newest vehicle in the network, having started operations in 2012. It is $37 \mathrm{~m}$ long with a 249-passenger capacity (including 68 seats) and has an integral low floor and six double doors per vehicle side.

\subsection{Data Collection}

The data used in this study were collected from the Opthor system, used by the operator. Opthor is an APC system measuring the number of passengers boarding and alighting at each station, on one hand, and the dwell time, as well as a number of other performance-related measures, on the other. Passengers are detected by an infrared system installed at each door of the vehicle. Dwell time is measured as the difference between the opening of the first door and the closing of the last door of the trainset. Since door operation is not forced by the driver, the value corresponds roughly to the duration of the passenger exchange. The operator has installed the system on a limited number of vehicles (of Alstom TFS and Bombardier Incentro class only) and runs frequent counts with it for various purposes.
The data employed here were collected during a count conducted between 5 September 2013 and 10 April 2014. As part of the count, the operator collected 134,500 valid entries from 124 weekdays and a total of 4900 runs. This sample corresponds to roughly $10 \%$ of the number of daily runs. Each data entry reports the total number of boarding and alighting passengers (as detected by the APC sensors), the number of passengers on board, and the corresponding dwell time at a station and for a given run.

Further filtering of the data was conducted to identify and limit the impact of extreme values. Specifically, only dwell time values between 1 and $120 \mathrm{~s}$ and passenger exchange volume values between 5 and 150 are considered. The reasoning behind this filtering is that the excluded values either lacked significance (less than $5 \mathrm{~s}$ of dwelling) for the scope of the research conducted, or were beyond the normal operational values. Given the high frequency of the lines, dwell times of over 2 min may be attributed to a system malfunction or schedule regulation strategies, and can consequently be excluded. On the other hand, the infrared technology used for passenger counting is less precise at high volumes of people. Finally, terminal stations are also omitted (both origin and destination), as the doors remain open at those during "turnback" and interior supervision, as well as the Commerce transfer station in the city centre, where holding strategies are applied. As such, following the filtering, a total of 118,957 observations are included in the analysis. 


\subsection{Analysis Methodology}

The analysis proceeds in two parts. The first part involves the investigation of dwell time patterns using a graphical method. Considering each line and direction separately, a set of heat maps of on-board passenger loading and dwell time are produced, allowing for the variation in these parameters to serve as a function of the location along the line and of the time of day to be observed. This enables the preliminary identification of causal relationships between dwell time and other relevant parameters, laying the groundwork for the second part of the analysis, which involves the use of inferential statistics to more formally identify these relationships.

In the second part, then, dwell time and its reliability are modelled using regression in order to derive explanatory statistical models. Dwell time is obtained directly from the field data, while dwell time reliability is estimated using aggregated values of dwell time for individual stations, lines and time periods across the entire network. Specifically, dwell time reliability is defined according to Kaparias et al. [38], where the lateness reliability index value is taken, calculated as the ratio of the mean to the 95th percentile dwell time.

The models for dwell time and reliability are estimated using multiple linear regression. The reason for this choice is that linear regression is one of the simplest techniques for modelling the relationship between a continuous dependent variable and several regressors that are thought to co-vary, and is very commonly employed in a wide range of transport applications. Both dwell time and reliability are continuous non-negative variables, and can be reasonably assumed to co-vary with the field data, which includes on-board passenger volumes, boarding and alighting passenger volumes, time of day, ranking and position of the stations along a line, and vehicle type.

Following Washington et al. [39], dwell time can be modelled as Eq. 2:

$Y_{i}=\beta_{0}+\beta_{j} X_{i j}+\varepsilon_{i}$

where $Y_{i}$ is the dwell time for station $i=1,2, \ldots$ of line $l$ for a specific vehicle run, $\beta_{0}$ is the constant term, $\beta_{j}$ denotes the coefficients to be estimated for $j=1,2, \ldots, \rho$ independent variables considered, and $\varepsilon_{i}$ is the disturbance term for station $i$. Similarly, dwell time reliability per tramway station can be modelled as in Eq. 2 with $Y_{i}$ representing the dwell time reliability of tramway station $i=1,2, \ldots$ of lines $l=1,2,3$ for all vehicle runs occurring within time period $H=1$ or $H=2$ or $H=3, \beta_{0}$ is the constant term, $\beta_{j}$ stands for the coefficients to be estimated for the $j=1,2, \ldots, \rho$ independent variables considered, and $\varepsilon_{i}$ is the disturbance term for tramway station $i$.
The error term of this type of regression model is independently and identically normally distributed with zero mean and constant variance. Furthermore, the functional form of the multiple linear regression in Eq. (2) assumes that the estimated parameters are the same for all observations. This assumption seems to hold, as no significant heterogeneity is observed.

\section{Results}

The results of the graphical and statistical analysis of dwell time of the tramway system are presented next.

\subsection{Graphical Analysis of Dwell Time}

Six heat map sets demonstrating the spatial and temporal variation in dwell time are produced and are shown in Figs. 2 and 3, each one corresponding to each of the two directions of each line. The first column on both figures lists the stations of the line in their order of occurrence in any run from bottom to top, while the first column of the heat maps shows the on-board passenger volume, expressed by means of crowding levels of service (LOS), as defined in TRB's TCQSM [8]. The second column then shows the average dwell time at each of the stations and time of day, classified into eight categories according to its duration.

A number of observations can be made from the heat maps. First and foremost, the average dwell time at Commerce station is considerably longer than anywhere else on the network. This is expected, as Commerce is the main interchange station, and holding strategies are applied there on all lines. A similar pattern is observed at Pirmil station, where Line 2 southbound merges with Line 3. This occurrence introduces bias in the data, and therefore justifies the decision to exclude the data from these two stations in further analysis.

Further interesting features can be extracted from the heat maps which enable the identification of parameters potentially affecting dwell time at stations. Namely, it can be observed that different dwell time patterns occur in the two directions of each line, which suggests that the line direction may have an influence on the dwell time. Also, the dwell time varies quite considerably throughout the day, with city-centre-bound runs closer to the start of the line experiencing higher dwell times in the morning peak and outskirts-bound runs closer to the end of the line facing higher dwell time in the evening peak. Stations located in the city centre, on the other hand, experience higher dwell times at all times and regardless of the line direction, and the same applies to stations located close to POIs, such as $\mathrm{P}+\mathrm{R}$ facilities, hospitals and educational establishments. 
Stations On-board crowding (passenger volume)

(a)

(b)
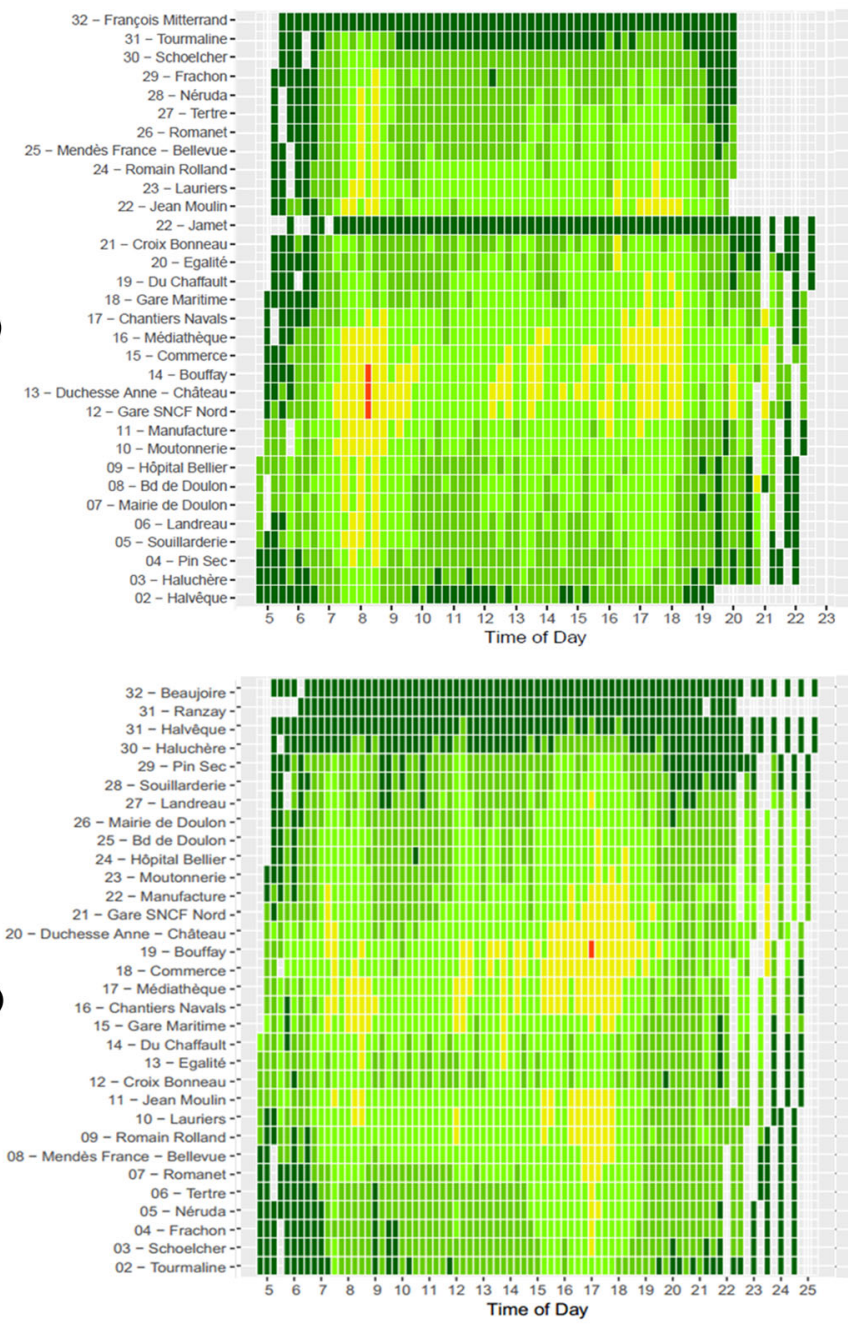

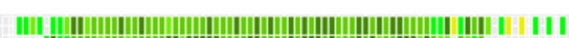

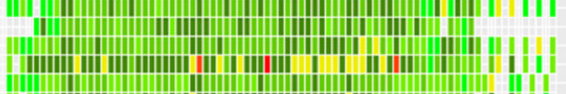

1. (n) ${ }_{n \rightarrow m}$ (n)

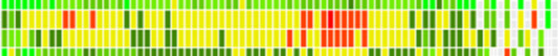

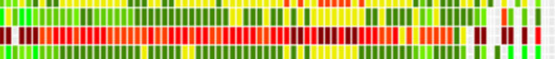

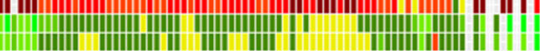
H. (n+m ,

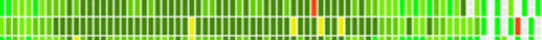
目国 . |

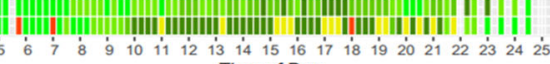
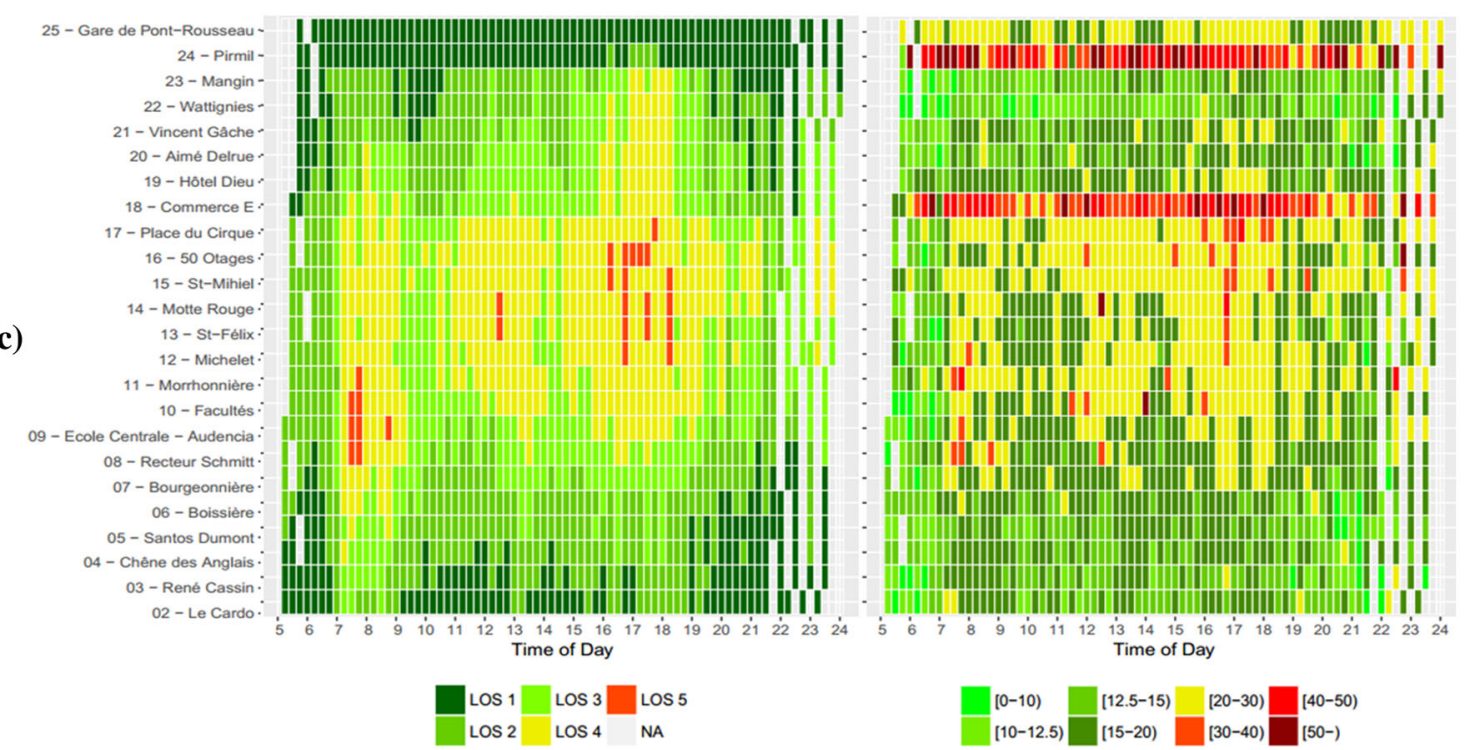

Fig. 2 Dwell time heat maps for each line and direction: a Line 1 westbound. b Line 1 eastbound. c Line 2 southbound 


\section{Stations On-board crowding (passenger volume)}

(a)

(b)

(c)
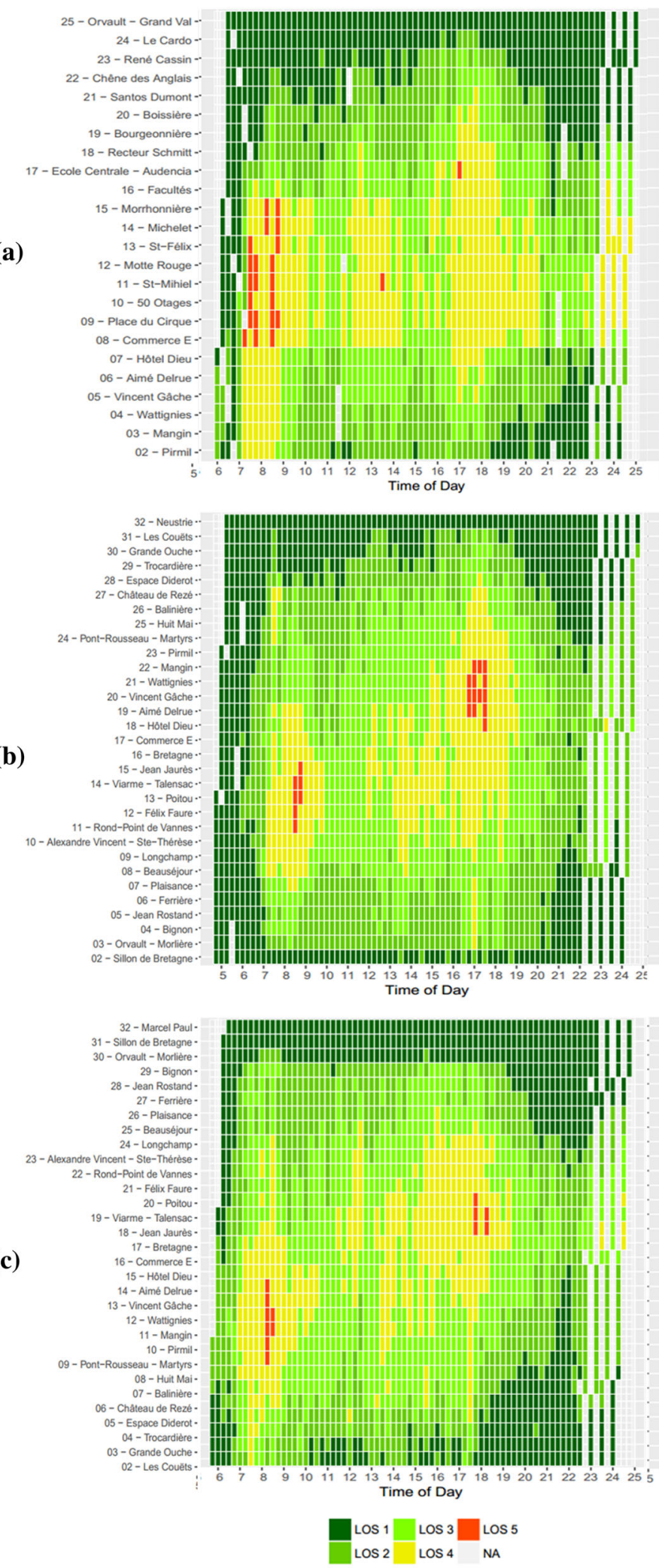

\section{Dwell time}
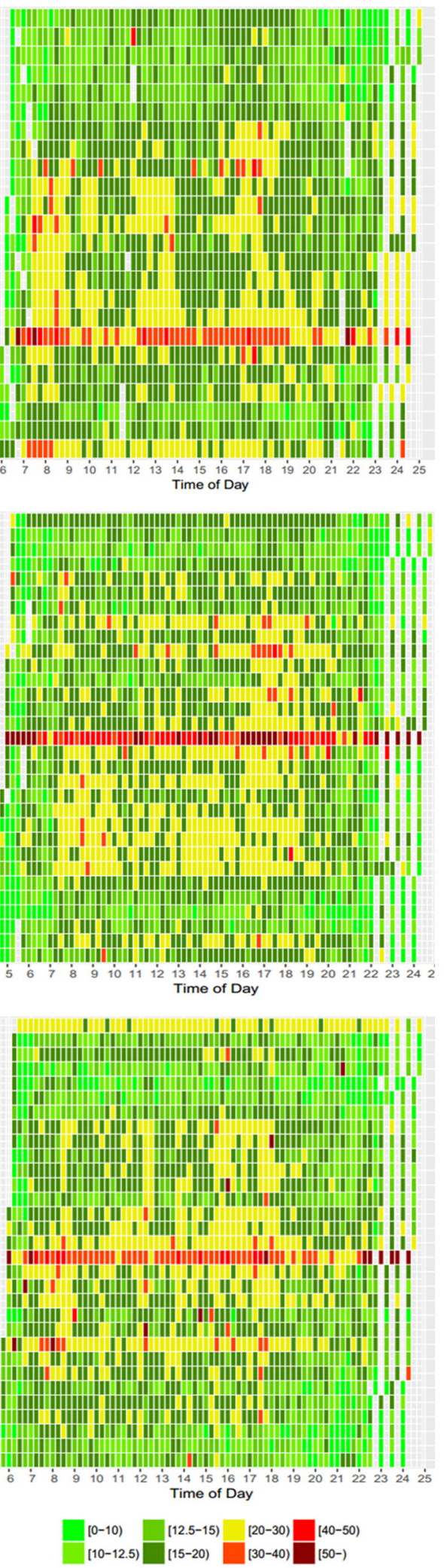

Fig. 3 Dwell time heat maps for each line and direction: a Line 2 northbound. b Line 3 southbound. c Line 3 northbound 
This suggests that the location (rank) of the station along the line and the time of day may have effects on dwell time.

Furthermore, it can be observed that there is an association between high on-board crowding levels and longer dwell times, but also that longer dwell times occur at stations and times where there are large changes (drops or increases) in on-board crowding. This suggests that the numbers of both on-board and of boarding and alighting passengers may have an influence. The potential causal relationships identified are next analysed more formally using inferential statistics.

\subsection{Statistical Modelling of Dwell Time}

Three multiple linear regression models are used to model dwell time from the field observational data, i.e. one per tramway line. The descriptive statistics of the models and the variables included in the regression are shown in Table 1, where it can be seen that the regression is based on large numbers of observations. Dwell time is modelled against the following variables, whose selection rationale is outlined in the previous subsection: the direction of the line ("LINE", categorical), the location of the station in the city centre ("CBD", binary), the time of day ("HOUR", categorical), the number of on-board passengers upon arrival at the station ("ONBO", continuous), the number of passengers boarding and alighting ("BNA", continuous), and the location of the station near a POI, a transport hub or a $\mathrm{P}+\mathrm{R}$ facility ("POI", binary). In addition, the influence of the vehicle type on the dwell time is examined ("VEH", categorical).

Ordinary least squares (OLS) regression is used to specify the models through the LIMDEP statistical software package, and the estimation results are shown in Table 2. The statistics of the model fitting are shown in part (a) of Table 2. As can be seen, the models achieve $R$ squared values of between 0.26 and 0.32 , which initially suggests that they may not be very good fits of the data. However, it should be considered here that the models are based on a very large number of observations, which increases the overall variability in the dataset, and hence makes it more difficult to achieve an accurate prediction. Also, it should be noted that the dependent variable takes generally small values, thus making the goodness of fit less significant, in the sense that prediction errors of a few seconds may appear as large from a statistical perspective, but are rather minor from a practical perspective. It can be concluded, therefore, that the models are acceptable fits.

The regression coefficients for each of the parameters included are then shown in part (b) of Table 2. Variables that are not statistically significant at the 0.05 level are omitted, and so all estimated parameters included in the final models are statistically significant. Elasticities are estimated for all continuous variables to assess the sensitivity of dwell time with respect to changes in the regressors.

Looking at the coefficients for each of the parameters, it can be first seen that the variable "LINE" has a statistically significant impact on dwell time at the 0.05 level. This finding indicates that different directions of the same line have different characteristics. In particular, longer dwell times are observed on the eastbound direction of Line 1 (compared with the westbound), on the northbound direction of Line 2 (compared with the southbound), and on the northbound direction of Line 3 (compared with the southbound). This can be explained by the smoother distribution of afternoon peaks relative to morning peaks, or by the local characteristics of the stations on each direction given that frequencies are equal. It is an important finding, indicating that future research efforts should consider the two directions of the same line separately when investigating dwell time variations.

It can then be seen that the variable "HOUR" is statistically significant for Lines 1 and 3 only. For Line 1, increased values of "HOUR" (that indicate moving towards peak hours) lead to higher dwell times. This intuitive finding supports the generally accepted idea that a larger number of passengers results in longer stopping. However, this is not the case for Line 3, where the opposite is observed. This may be due to increased frequency during the peaks in order to accommodate increasing demand. This analysis is important, as it allows for direct recommendations to the operators on whether planned increased frequencies during peak hours can sufficiently respond to the increased demand without a loss in levels of service. Along the same line of reasoning, it can be deduced that the variable "HOUR" is not significant for Line 2, as the frequency increase outbalances the extra passengers during the peak hours.

Furthermore, the volume of passengers on board upon arrival ("ONBO") and the volume of boarding and alighting passengers ("BNA") are statistically significant in all models. This is an intuitive result, as higher levels of passenger numbers, both boarding/alighting and staying aboard the vehicle, are likely to cause longer dwell times. As in-vehicle congestion can make access to the doors difficult for alighting passengers and also impede the boarding of new passengers, this can result in longer stops. It should be noted here that for a given vehicle run, the coefficient of "BNA" and the constant term of the model can be used to approximate the parameters of Eq. 1 for different on-board passenger levels.

In addition, the vehicle type ("VEH") has statistically significant effects on the dwell time for the Line 1 and Line 3 models. Specifically, it appears that there is a strong 
Table 1 Descriptive statistics of the dwell time regression models

\begin{tabular}{|c|c|c|c|}
\hline Variable & Type & Definition & Descriptive statistics \\
\hline \multicolumn{3}{|c|}{ Line 1: F. Mitterrand/Jamet-Ranzay/Beaujoire } & Number of observations $=65,535$ \\
\hline DT & Continuous & Dwell time (s) & $\begin{array}{l}\text { Min }=6.0 ; \operatorname{Max}=119.0 \\
\text { Mean }=17.0 ; \mathrm{SD}=8.6\end{array}$ \\
\hline LINE & Categorical & $\begin{array}{l}=1 \text { if eastbound; } \\
=2 \text { if westbound }\end{array}$ & $\begin{array}{l}F(1)=0.47 \\
F(2)=0.53\end{array}$ \\
\hline CBD & Binary & $\begin{array}{l}=1 \text { if station within } \mathrm{CBD} \\
=0 \text { otherwise }\end{array}$ & $F(1)=0.41$ \\
\hline HOUR & Categorical & $\begin{array}{l}=1 \text { if } 4 \mathrm{~h} 00-6 \mathrm{~h} 59 \\
=2 \text { if } 9 \mathrm{~h} 00-15 \mathrm{~h} 59 \text { or } 19 \mathrm{~h} 00-19 \mathrm{~h} 30 \\
=3 \text { if } 7 \mathrm{~h} 30-8: 59 \text { or } 16 \mathrm{~h} 00-18 \mathrm{~h} 59\end{array}$ & $\begin{array}{l}F(1)=0.10 \\
F(2)=0.57 \\
F(3)=0.33\end{array}$ \\
\hline ONBO & Continuous & Number of onboard passengers upon arrival & $\begin{array}{l}\text { Min }=0.0 ; \operatorname{Max}=236.0 \\
\text { Mean }=50.5 ; \mathrm{SD}=34.7\end{array}$ \\
\hline BNA & Continuous & Number of passengers boarding and alighting per station & $\begin{array}{l}\text { Min }=1.0 ; \operatorname{Max}=148.0 \\
\text { Mean }=14.7 ; \mathrm{SD}=14.4\end{array}$ \\
\hline VEH & Categorical & $\begin{array}{l}=1 \text { if vehicle type is Bombardier } \\
=2 \text { if vehicle type is Alstom }\end{array}$ & $\begin{array}{l}F(1)=0.51 \\
F(2)=0.49\end{array}$ \\
\hline POI & Binary & $\begin{array}{l}=1 \text { if station is a POI, has } \mathrm{P}+\mathrm{R} \text {, or is a transfer hub; } \\
=0 \text { otherwise }\end{array}$ & $F(1)=0.19$ \\
\hline Variable & Type & Definition & Descriptive statistics \\
\hline \multicolumn{3}{|c|}{ Line 2: Orvault Grand Val-Gare de Pont Rousseau } & Number of observations $=26,680$ \\
\hline DT & Continuous & Dwell time (s) & $\begin{array}{l}\text { Min }=6.0 ; \operatorname{Max}=120.0 \\
\text { Mean }=18.7 ; \mathrm{SD}=9.0\end{array}$ \\
\hline LINE & Categorical & $\begin{array}{l}=1 \text { if southbound; } \\
=2 \text { if northbound }\end{array}$ & $\begin{array}{l}F(1)=0.52 \\
F(2)=0.48\end{array}$ \\
\hline CBD & Binary & $\begin{array}{l}=1 \text { if station within } \mathrm{CBD} \\
=0 \text { otherwise }\end{array}$ & $F(1)=0.41$ \\
\hline HOUR & Categorical & $\begin{array}{l}=1 \text { if } 4 \mathrm{~h} 00-6 \mathrm{~h} 59 \\
=2 \text { if } 9 \mathrm{~h} 00-15 \mathrm{~h} 59 \text { or } 19 \mathrm{~h} 00-19 \mathrm{~h} 30 \\
=3 \text { if } 7 \mathrm{~h} 30-8: 59 \text { or } 16 \mathrm{~h} 00-18 \mathrm{~h} 59\end{array}$ & $\begin{array}{l}F(1)=0.04 \\
F(2)=0.63 \\
F(3)=0.33\end{array}$ \\
\hline ONBO & Continuous & Number of onboard passengers upon arrival & $\begin{array}{l}\text { Min }=0.0 ; \text { Max }=268.0 \\
\text { Mean }=58.34 ; \mathrm{SD}=43.83\end{array}$ \\
\hline BNA & Continuous & Number of passengers boarding and alighting per station & $\begin{array}{l}\text { Min }=1.0 ; \operatorname{Max}=150.0 \\
\text { Mean }=18.1 ; \operatorname{SD}=16.1\end{array}$ \\
\hline VEH & Categorical & $\begin{array}{l}=1 \text { if vehicle type is Bombardier } \\
=2 \text { if vehicle type is Alstom }\end{array}$ & $\begin{array}{l}F(1)=0.00 \\
F(2)=1.00\end{array}$ \\
\hline POI & Binary & $\begin{array}{l}=1 \text { if station is a POI, has } \mathrm{P}+\mathrm{R} \text {, or is a transfer hub; } \\
=0 \text { otherwise }\end{array}$ & $F(1)=0.23$ \\
\hline
\end{tabular}


Table 1 continued

\begin{tabular}{|c|c|c|c|}
\hline Variable & Type & Definition & Descriptive statistics \\
\hline \multicolumn{3}{|c|}{ Line 3: Marcel Paul-Neustrie } & Number of observations $=25,942$ \\
\hline DT & Continuous & Dwell time (s) & $\begin{array}{l}\text { Min }=6.0 ; \operatorname{Max}=116.0 \\
\text { Mean }=17.7 ; \mathrm{SD}=7.7\end{array}$ \\
\hline LINE & Categorical & $\begin{array}{l}=1 \text { if southbound; } \\
=2 \text { if northbound }\end{array}$ & $\begin{array}{l}F(1)=0.50 \\
F(2)=0.50\end{array}$ \\
\hline CBD & Binary & $\begin{array}{l}=1 \text { if station within CBD; } \\
=0 \text { otherwise }\end{array}$ & $F(1)=0.28$ \\
\hline HOUR & Categorical & $\begin{array}{l}=1 \text { if } 4 \mathrm{~h} 00-6 \mathrm{~h} 59 \\
=2 \text { if } 9 \mathrm{~h} 00-15 \mathrm{~h} 59 \text { or } 19 \mathrm{~h} 00-19 \mathrm{~h} 30 \\
=3 \text { if } 7 \mathrm{~h} 30-8: 59 \text { or } 16 \mathrm{~h} 00-18 \mathrm{~h} 59\end{array}$ & $\begin{array}{l}F(1)=0.08 \\
F(2)=0.59 \\
F(3)=0.33\end{array}$ \\
\hline ONBO & Continuous & Number of onboard passengers upon arrival & $\begin{array}{l}\text { Min }=0.0 ; \operatorname{Max}=232.0 \\
\text { Mean }=50.7 ; \mathrm{SD}=36.6\end{array}$ \\
\hline BNA & Continuous & Number of passengers boarding and alighting per station & $\begin{array}{l}\text { Min }=1.0 ; \operatorname{Max}=135.0 \\
\text { Mean }=13.8 ; \mathrm{SD}=12.8\end{array}$ \\
\hline VEH & Categorical & $\begin{array}{l}=1 \text { if vehicle type is Bombardier } \\
=2 \text { if vehicle type is Alstom }\end{array}$ & $\begin{array}{l}F(1)=0.14 \\
F(2)=0.86\end{array}$ \\
\hline POI & Binary & $=1$ if station is a POI, has $\mathrm{P}+\mathrm{R}$, or is a transfer hub; & $F(1)=0.30$ \\
\hline
\end{tabular}

Table 2 Model estimation results for dwell time

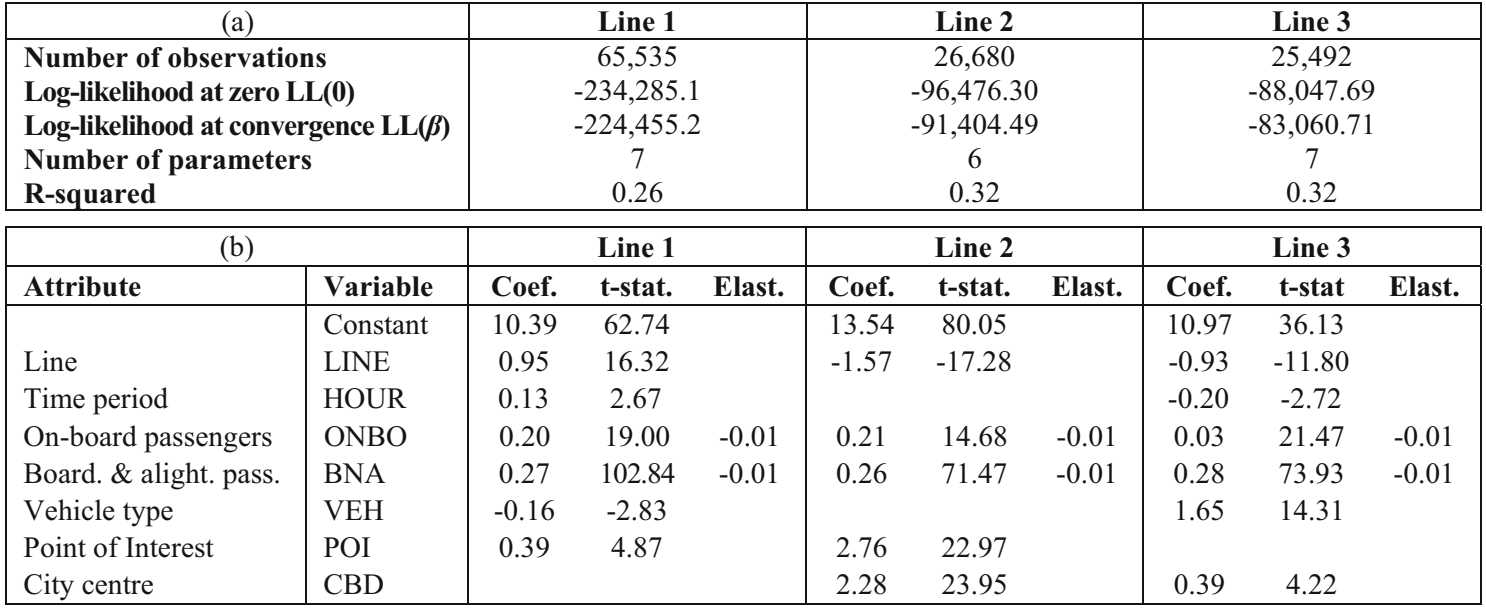

association of new low-floor vehicles (Bombardier) with shorter dwell times on Line 3, but also a weak association with longer dwell times on Line 1 . An explanation could be the fact that Line 1 has a greater proportion of segregated track and dedicated rail-type stations (especially at each eastern section) than Line 3 , already providing an important facility for the boarding and alighting of passengers, such that the impact of the vehicle type is less evident. Conversely, many more stations along Line 3 are designed conventionally, and at such locations the new low-floor vehicles do much more to facilitate the boarding and alighting process, and hence have a positive impact in reducing dwell time.

Moreover, the "POI" and "CBD" variables appear to have significant effects on the dwell time, as dwell times at stations located in the city centre and at stations serving one or more POIs are generally longer than those at other stations. Both variables have strong significant effects on dwell time along Line 2 stations, while weaker effects are observed in the other two lines, with "POI" being significant only in Line 1 and "CBD" only in Line 3. This is a sensible result, as stations located in the city centre and 
close to POIs are usually associated with larger passenger volumes, which increases dwell time. It should be noted, however, that some of the effect of these variables is likely to be confounded with that of the "BNA" variable, and further analysis of the two- and three-way interaction effects, in addition to the main effects, is required to shed light on these. This, however, is beyond the scope of the present study.

Finally, the elasticity analysis of the continuous variables shows very low sensitivity of dwell time to boarding and alighting volumes. This can be explained by the high value of the constant term that is observed in all models and corresponds to a fixed minimum dwell time that cannot be under-passed. As such, for low passenger volumes there is very little or no impact on dwell time. This is sensible, as the vehicles employed have six large doors that allow for many simultaneous boarders and alighters, and only little impact on dwell time is expected.

\subsection{Statistical Modelling of Dwell Time Reliability}

A multiple linear regression model is used to model dwell time reliability and field observational data for all three lines together. The descriptive statistics of the model and the variables included in the regression are shown in Table 3; as can be seen, the number of observations is now significantly lower due to the aggregation of the data to establish the reliability values. Dwell time reliability is modelled against the following variables: the location of the station along the line ("RANK", ordered); the time of day ("HOUR", categorical); the location of the station near a POI, a transport hub or a P+R facility ("POI", binary); the average number of on-board passengers upon arrival at the station ("AVONBO", continuous); the average number of passengers boarding and alighting ("AVBNA", continuous); and six variables denoting the line serving the station and the direction ("LINE1A", "LINE 1B", "LINE2A", "LINE2B", "LINE3A" and "LINE3B"). It should be noted here that some stations are served by both Line 2 and Line 3.

Table 3 Descriptive statistics of the dwell time reliability regression model

\begin{tabular}{|c|c|c|c|}
\hline $\begin{array}{l}\text { Variable } \\
\text { name }\end{array}$ & Type & Definition & Descriptive statistics \\
\hline SREL & Continuous & Reliability $=$ MeanDT/95percentile of DT per station $(\mathrm{P})$ and hour of the day $(\mathrm{H})$ & $\begin{array}{l}\text { Number of observations }=354 \\
\text { Min }=0.38 ; \operatorname{Max}=0.8 \\
\text { Mean }=0.6 ; \mathrm{SD}=0.1\end{array}$ \\
\hline RANK & Ordered & $\begin{array}{l}=1 \text { if the station is on first third of the line; } \\
=2 \text { if station is on the second third of the line; } \\
=3 \text { if station is on the third part of the line }\end{array}$ & $\begin{array}{l}F(1)=0.27 \\
F(2)=0.31 \\
F(3)=0.42\end{array}$ \\
\hline HOUR & Categorical & $\begin{array}{l}=1 \text { if } 4 \mathrm{~h} 00-6 \mathrm{~h} 59 \\
=2 \text { if } 9 \mathrm{~h} 00-15 \mathrm{~h} 59 \text { or } 19 \mathrm{~h} 00-19 \mathrm{~h} 30 \\
=3 \text { if } 7 \mathrm{~h} 30-8: 59 \text { or } 16 \mathrm{~h} 00-18 \mathrm{~h} 59\end{array}$ & $\begin{array}{l}F(1)=0.33 \\
F(2)=0.33 \\
F(3)=0.33\end{array}$ \\
\hline POI & Binary & $\begin{array}{l}=1 \text { if station is a point of interest, has a } \mathrm{P} \& \mathrm{R} \text { facility or is a transfer hub; } \\
=0 \text { otherwise }\end{array}$ & $F(1)=0.27$ \\
\hline AVONBO & Continuous & Average number of onboard passengers upon arrival at station $\mathrm{P}$ and over period $\mathrm{H}$ & $\begin{array}{l}\text { Min }=1.4 ; \operatorname{Max}=116.8 \\
\text { Mean }=47.5 ; \mathrm{SD}=24.3\end{array}$ \\
\hline AVBNA & Continuous & Average number of boarding and alighting passengers at station $\mathrm{P}$ and over period $\mathrm{H}$ & $\begin{array}{l}\text { Min }=1.3 ; \operatorname{Max}=51.1 \\
\text { Mean }=13.2 ; \mathrm{SD}=9.0\end{array}$ \\
\hline LINE1A & Binary & $\begin{array}{l}=1 \text { if station served by line } 1 \text { eastbound; } \\
=0 \text { otherwise }\end{array}$ & $F(1)=0.25$ \\
\hline LINE1R & Binary & $\begin{array}{l}=1 \text { if station served by line } 1 \text { westbound; } \\
=0 \text { otherwise }\end{array}$ & $F(1)=0.25$ \\
\hline LINE2A & Binary & $\begin{array}{l}=1 \text { if station served by line } 2 \text { southbound; } \\
=0 \text { otherwise }\end{array}$ & $F(1)=0.20$ \\
\hline LINE2R & Binary & $\begin{array}{l}=1 \text { if station served by line } 2 \text { northbound; } \\
=0 \text { otherwise }\end{array}$ & $F(1)=0.19$ \\
\hline LINE3A & Binary & $\begin{array}{l}=1 \text { if station served by line } 3 \text { southbound; } \\
=0 \text { otherwise }\end{array}$ & $F(1)=0.25$ \\
\hline LINE3R & Binary & $\begin{array}{l}=1 \text { if station served by line } 3 \text { northbound; } \\
=0 \text { otherwise }\end{array}$ & $F(1)=0.25$ \\
\hline
\end{tabular}


Table 4 Model estimation results for dwell time reliability

\begin{tabular}{|l|c|}
\hline \multicolumn{2}{|c|}{ (a) } \\
\hline Number of observations & 348 \\
Log-likelihood at zero LL(0) & 526.3615 \\
Log-likelihood at convergence LL( $\boldsymbol{\beta})$ & 507.5840 \\
Number of parameters & 6 \\
R-squared & 0.1 \\
\hline
\end{tabular}

\begin{tabular}{|l|l|ccc|}
\hline \multicolumn{5}{|c|}{ (b) } \\
\hline Attribute & Variable & Coef. & t-stat. & Elast. \\
\hline & Constant & 0.6478 & 66.8740 & \\
Line 1 eastbound & LINE1A & -0.0139 & -1.9200 & \\
Line 1 westbound & LINE1R & -0.0130 & -1.8050 & \\
Location along line & RANK & -0.0100 & -2.7730 & \\
City centre & CBD & -0.0184 & -2.8470 & \\
Average board. \& alight. pass. & AVBNA & -0.0010 & -2.9710 & 0.17 \\
\hline
\end{tabular}

OLS regression is used to specify the model through the LIMDEP statistical software package, and the estimation results are shown in Table 4. The statistics of the fitting of the models are shown in part (a) of Table 4. As can be seen, the model achieves an $R$-squared value of 0.1 , which, again, initially appears to be a not particularly strong fit. However, similarly to the dwell time model and even more manifestly, the dependent variable here takes very small values (between 0 and 1 in theory, and between 0.38 and 0.8 in practice), such that prediction errors of a few decimal points may appear as large from a statistical perspective, but are still minor from a practical perspective. It can be, hence, concluded that the model is an acceptable fit.

The regression coefficients for each of the parameters included are then shown in part (b) of Table 4. Variables that are not statistically significant at the 0.05 level are omitted, and hence all estimated parameters included in the final models are statistically significant. Elasticities are estimated for the one continuous variable to assess the sensitivity of dwell time reliability.

Looking at the coefficients for each of the parameters, it can first be observed that their values are low. This can be partly explained, again, by the fact that reliability takes values between 0 and 1 in theory, but a further reason could be that there is little variation in reliability values around the network, which influences the model's explanatory power. Starting with the variables' coefficients, the variables "LINE1A" and "LINE1B" have statistically significant negative impacts on dwell time reliability at the 0.05 level. This finding indicates that dwell time reliability is likely to be lower at the stations of Line 1 than the other lines. It can be attributed on one hand to the structure of Line 1, which has branches at the extremities, and on the other hand to the higher number of facilities (rail and regional transfer points) of Line 1 , which add up to a random demand within the same time period.

It can then be seen that the variable "RANK" has a negative statistically significant effect on dwell time reliability, such that stations located further away from the start along the line appear to experience lower dwell time reliability. This is a sensible result, as variable delays along a line may result in higher numbers of passengers accumulating at stations, which may lead to more or less time needed for boarding and alighting, and hence greater variability in the dwell time. This, however, would be less evident close to the starting terminal, as the delay accumulation is likely to be small and would not be expected to rise until further down.

Furthermore, the "CBD" and "AVBNA" variables appear to have significant negative effects on dwell time reliability, such that the reliability at stations located in the city centre and at stations with high boarding/alighting passenger volumes is generally lower than at other stations. This is again, a sensible result, which is also in line with the findings of the dwell time modelling. Specifically, stations located in the city centre are usually associated with larger passenger volumes, which increases dwell time. Given, however, that these stations are also typically located far from the starting terminal of the line, they are also prone to delays, which affects the accumulation of passenger volumes and thus may result in variability in the time needed for boarding/alighting. Consequently, dwell time reliability is likely to be lower at these locations. Still, just as in the case of dwell time, it should be noted that some of the effects of the "RANK", "CBD" and "AVBNA" variables are likely to be confounded by one another, and further analysis of the two- and three-way interaction effects, in addition to the main effects, is needed to shed light on these. This, however, is beyond the scope of the present study and could be investigated as part of future research studies.

Finally, last but not least, an elasticity of boarding and alighting volumes over dwell time reliability of 0.17 is reported, if all other variables are kept constant. This finding confirms that at a given station and during a given period of time, volumes become important and may have a considerable impact on dwell time variability.

\section{Conclusions}

The aim of this study was to investigate the topic of vehicle dwell time at urban light rail facilities and to identify cause-and-effect relationships. Using APC data from the tramway system of the city of Nantes collected over a period of 1 year, graphical and statistical analyses were carried out in order to identify how dwell time and its reliability may be influenced by a number of attributes. The results confirm that, as conjectured, boarding and alighting passenger volumes, as well as on-board passenger loading, have a significant impact on both dwell time and reliability. 
Additional significant effects on dwell time are found from the vehicle type (low- or high-floor), from the time of day (peak, off-peak, inter-peak), and from the location of the station (city centre, close to POIs). An interesting result in this context is also the lack of operational symmetry between the directions of a line, in that stations located at different directions of the same line exhibit different dwell time trends. A further effect detected is that of the location of the station along the line (distance from the starting terminal) on reliability, with stations located far downstream generally experiencing greater dwell time variability.

As with every study, naturally, there are some limitations. For instance, while the proposed models offer useful insight into how total passenger boarding and alighting throughput affects dwell time and reliability, they are not able to explore the impact of the boarding and alighting processes separately. Indeed, the TCQSM dwell time model (Eq. 1) assumes that boarding and alighting are two completely separate processes, with the former strictly following the latter, which suggests that different effects may be present. However, from the authors' observations of the Nantes tramway, it was found that the width of the doors and the low levels of passenger flows during off-peak times often allow for simultaneous boarding and alighting, while the observed distribution of passengers along the platform is rather uniform. As such, it is difficult to single out the dynamics of passenger mingling at the proximity of the doors, and a different modelling approach (e.g. social force model [40]) would be required to analyse them in detail, which is beyond the scope of this study. This could be explored as part of future research on the topic.

Furthermore, a limitation of the proposed multiple linear regression models can be identified in the justified but still objectively low R-squared values when estimated by OLS regression. Additional comparative analysis by the authors of the predictive power of the proposed models against the operator's current prediction technique using a separate subset of the data, however, provides important insight into this issue. As it turns out, the operator's own dwell time estimation practice for scheduling purposes is to assume fixed values that apply to all days and times for the entirety of the line; these values are, naturally, rather simplistic, as they do not consider passenger flow oscillations or station particularities. A comparison of the root mean square error (RMSE) between the observed dwell times and the predicted values using the proposed models, and between observed and scheduled dwell times (using the operator's fixed values), found that on average, an improvement of $52-65 \%$ can be achieved. This means that even though it is clear that the accuracy of the proposed models can be further improved, the models still enable better estimation than current practice, and could be easily integrated into the scheduling process.

It can be concluded, therefore, that the findings of the present study could be effectively used to devise solutions for minimising the important source of time loss that is dwell time. But while the study has shed some light on the determinants of the dwell time of tramway systems, research in this direction continues. Further work by the authors in the first instance will concentrate on exploring the findings in greater depth, with a view to further improving and refining the proposed models. For instance, a more detailed investigation is planned to examine the factors that lead to non-symmetrical operations, aiming to provide useful insight for efficient configurations of light rail lines. In these terms, a comprehensive investigation of different station configurations will be carried out to determine their impact on the dwell time and its reliability. Similarly, research will be conducted to more comprehensively explore the differences in dwell time and reliability patterns between the different lines and different times in order to relate it to additional line characteristics (e.g. differences between trunk or branch sections) as well as other factors such as weather conditions. A similar analysis will be undertaken for other tramway and for bus networks in order to compare and confirm the significance of non-site-specific determinants.

The study's findings, however, also provide inspiration for further research on the topic, beyond the immediate improvement and refinement of the proposed models. For instance, it is planned to extend the research in the direction of real-time operations, and more specifically in the short-term prediction of on-board passenger loads and corresponding dwell times, which could serve as input to advanced transit assignment models (e.g. [41-43]). A key challenge in this respect lies in the fact that typically only a small subset of a fleet's vehicles are equipped with APC sensors, which means that operators must rely on partial information, and future work is planned to explore the implications of this challenge and to devise ways to address it.

Acknowledgements The authors would like to thank Semitan for supplying the data used in this study, and in particular the "Direction de la Performance et de l'Innovation" of Semitan for their assistance in analysing the Winter 2014 Opthor dataset.

Open Access This article is distributed under the terms of the Creative Commons Attribution 4.0 International License (http://crea tivecommons.org/licenses/by/4.0/), which permits unrestricted use, distribution, and reproduction in any medium, provided you give appropriate credit to the original author(s) and the source, provide a link to the Creative Commons license, and indicate if changes were made. 
Authors' Contributions The authors confirm contributions to the paper as follows: study conception and design: ZC, EC, IK; literature search and review: EC, IK; data collection: ZC, EC; analysis and interpretation: ZC, EC, IK; manuscript preparation: IK. All authors reviewed the results and approved the final version of the manuscript.

Availability of Data and Materials The raw dataset used in this study is the property of Semitan and therefore cannot be shared. The authors would be happy to supply aggregated/processed versions of the data to interested parties upon request.

\section{Compliance with Ethical Standards}

Conflict of interest The authors declare that they have no conflict of interest.

\section{References}

1. Wardrop JG (1952) Some theoretical aspects of road traffic research. Proc Inst Civ Eng 1:325-378

2. Jackson WB, Jucker JV (1981) An empirical study of travel time variability and travel choice behaviour. Transp Sci 16:460-475

3. Adbel-Aty A, Kitamura R, Jovanis PP (1995) Investigating the effect of travel time variability on route choice using repeated measurement stated preference data. Transp Res Rec 1493:39-45

4. Lam TC, Small KA (2001) The value of time and reliability: measurement from a value pricing experiment. Transp Res Part E 37:231-251

5. Turnquist M, Bowman L (1980) The effects of network structure on reliability of transit service. Transp Res Part B 14:79-86

6. Shang P, Li R, Liu Z, Xian K, Guo J (2018) Timetable synchronization and optimization considering passenger demand in an urban subway network. Transp Res Rec 2672:243-254

7. Shang P, Li R, Liu Z, Yang L, Wang Y (2018) Equity-oriented skip-stopping schedule optimization in an oversaturated urban rail transit network. Transp Res Part C 89:321-343

8. Transportation Research Board (2013) Transit capacity and quality of service manual, 3rd edn. Transportation Research Board, Washington, DC

9. Szplett D, Wirasinghe SC (1984) An investigation of passenger interchange and train standing time at LRT stations: alighting, boarding and platform distribution of passengers. J Adv Transp $18: 1-12$

10. Wirasinghe SC, Szplett D (1984) An investigation of passenger interchange and train standing time at LRT stations: estimation of standing time. J Adv Transp 18:13-24

11. Oliveira L, Fox C, Birrell S, Cain R (2019) Analysing passengers' behaviours when boarding trains to improve rail infrastructure and technology. Robot Comput Integr Manuf 57:282-291

12. Fritz M (1983) Effect of crowding on light rail passenger boarding times. Transp Res Rec 908:43-50

13. Lin T-M, Wilson NHM (1992) Dwell time relationships for light rail systems. Transp Res Rec 1361:287-295

14. Cornet S, Buisson C, Ramond F, Bouvarel P (2019) Methods for quantitative assessment of passenger flow influence on train dwell time in dense traffic areas. Transp Res Part C 106:345-359

15. Currie G, Delbosc A, Harrison S, Sarvi M (2013) Impact of crowding on streetcar dwell time. Transp Res Rec 2353:100-106

16. Daamen W, Lee YC, Wiggenraad P (2008) Boarding and alighting experiments: an overview of the set up and performance and some preliminary results. Transp Res Rec 2042:71-81

17. Fernandez R, Zegers P, Weber G, Tyler N (2010) Influence of platform height, door width and fare collection on bus dwell time: laboratory evidence from Santiago de Chile. Transp Res Rec 2143:59-66

18. Milkovits M (2008) Modeling the factors affecting bus stop dwell time. Transp Res Rec 2072:125-130

19. Weston JG (1989) Train service model—technical guide. London Underground Operational Research Note 89/18

20. Harris NG, Anderson RJ (2007) An international comparison of urban rail boarding and alighting rates. Proc Inst Mech Eng Part F J Rail Rapid Transit 221:521-526

21. Harris NG (2005) Train boarding and alighting rates at high passenger loads. J Adv Transp 40:249-263

22. Zhang Q, Han B, Li D (2008) Modeling and simulation of passenger alighting and boarding movement in Beijing metro stations. Transp Res Part C 16:635-649

23. Noland RB, Small KA (1995) Travel-time uncertainty, departure time choice, and the cost of morning commutes. Transp Res Rec 1493:150-158

24. Bates J, Black I, Fearon J, Gilliam C, Porter S (2002) Supply models for use in modelling the variability of journey times on the highway network. In: European Transport Conference 2002, Cambridge, UK

25. Liu HX, Recker W, Chen A (2004) Uncovering the contribution of travel time reliability to dynamic route choice using real-time loop data. Transp Res Part A 38:435-453

26. Bates J, Polak JW, Jones P, Cook A (2001) The valuation of reliability for personal travel. Transp Res Part E 37:191-229

27. Noland RB, Polak JW (2002) Travel time variability: a review of theoretical and empirical issues. Transp Rev 22:39-54

28. Bell MGH, Iida Y (1997) Transportation network analysis. Wiley, Chichester

29. Chen K, Yu L, Guo J, Wen H (2007) Characteristics analysis of road network reliability in Beijing based on the data logs from taxis. In: 86th annual meeting of the transportation research board, Washington, DC, USA

30. Eleftheriadou L, Cui X (2007) A framework for defining and estimating travel time reliability. In: 86th annual meeting of the transportation research board, Washington, DC, USA

31. Polus A (1979) A study of travel time and reliability on arterial routes. Transportation 8:141-151

32. Dandy GC, McBean EA (1984) Variability of individual travel time components. ASCE J Transp Eng 110:340-357

33. van Lint JWC, van Zuylen HJ (2005) Monitoring and predicting freeway travel time reliability-using width and skew of day-today travel time distribution. Transp Res Rec 1917:54-62

34. van Oort N (2011) Service reliability and urban public transport design. Ph.D. Thesis, TRAIL Research School, Delft, Netherlands

35. Furth PG, Muller THJ (2007) Service reliability and optimal running time schedules. Transp Res Rec 2034:55-61

36. Lomax T, Turner S, Margiotta R (2001) Monitoring urban roadways in 2000: Using archived operations data for reliability and mobility measurement. In: FHWA-OP-02-029, Federal Highway Administration-Operations Core Business Unit, USA

37. Lomax T, Schrank D, Turner S and Margiotta R (2003) Selecting travel reliability measures. 474360-1. Texas Transportation Institute, USA

38. Kaparias I, Bell MGH, Belzner H (2008) A new measure of travel time reliability for in-vehicle navigation systems. J Intell Transp Syst 12:202-211

39. Washington SP, Karlaftis MG, Mannering FL (2010) Statistical and econometric methods for transportation data analysis, 2nd edn. CRC, Boca Raton

40. Helbing D, Molnar P (1995) Social force model for pedestrian dynamics. Phys Rev E 51:4282-4286 
41. Trozzi V, Gentile G, Bell MGH, Kaparias I (2013) Dynamic user equilibrium in public transport networks with passenger congestion and hyperpaths. Transp Res Part B 57:266-285

42. Leurent $F$, Chandakas E, Poulhès A (2014) A traffic assignment model for passenger transit on a capacitated network: bi-layer framework, line sub-models and large-scale application. Transp Res Part C 47:3-27
43. Shang P, Li R, Guo J, Xian K, Zhou X (2019) Integrating Lagrangian and Eulerian observations for passenger flow state estimation in urban rail transit network: a space-time-state hyper network-based assignment approach. Transp Res Part B 121:135-167 